\title{
Golpe de calor en lactantes
}

\author{
Dr. Ernesto Núñez M. ${ }^{1}$; Dra. Elizabeth Vega A. ${ }^{1}$; Dr. Alfonso Martínez M. ${ }^{2}$; \\ Dr. José Schalper P. 2
}

\section{Heatstroke in infants}

Four infants under one year of age presented with a clinical disorder characterized by acute onset of coma, convulsions, shock, hemorrage, hyper thermia, acidosis, leucocy tosis, hepatomegaly, dianhoea and alterations in liver and kidney function. Three patients died, and the survivor has severe neurological sequelae. At autopsy there was evidence of cerebral edema, focal gliosis, fatty focal infiltration of the liver, edema and hyperemia of the intestinal mucos with increased lymphatic follicles. This disorder is similar to descriptions of heatstroke in infants. It's close resemblance to the association of hemorrhagic shock and encephalopathy, that has been recently reported, is pointed out. It is proposed that they both correspond to the same entity. The prevalence in the masculine sex and the potential prevention are emphasized.

(Key words: Heatstroke, shock, hyperthermis, encephalopathy).

En 1979 , Bacon ${ }^{1}$ describió un síndrome clínico, en lactantes menores de 1 año, caracte-

1. Médico. Servicio de pediatría. Hospital Las Higueras, Talcahuano.

2. Médico. Servicio de anatomía patológica. Hospital Las Higueras, Talcahuano. rizado por fiebre alta, shock, convulsiones, alteraciones hemorrágicas y una encefalopatía catastrófica que condujo a la nuerte a 4 de los 5 pacientes observados, que el autor atribuyó al golpe de calor ${ }^{2}$. En los últimos 12 meses, hemos ingresado al hospital 4 lactantes con sintomas semejantes a las descritas por Bacon, de los que 3 
pacientes fallecieron. Presentamos sus antecedentes para llamar la atención sobre esta afección y su eventual prevención.

\section{Pacientes}

1. Lactante de 4 meses, masculino, comenzó con tos, coriza y anorexia cuatro dias antes de su ingreso, motivo por el que recibió ampicilina $125 \mathrm{mg}$ cada 6 horas. Ingresó inconciente, con convulsiones tónico clónicas generalizadas, temperatura rectal de $41,5^{\circ} \mathrm{C}$, hidratado, bregma normal, sin signos localizados de infección. Trata. do con diazepam endovenoso, fue necesario emplear dosis de $6 \mathrm{mg}$ para controlar las convulsiones. Luego recibió ampicilina $100 \mathrm{mg} \mathrm{x} \mathrm{kg} \times$ día endovenosa, gentanjicina $5 \mathrm{mg} x \mathrm{~kg} x$ dia intramuscular, e hidratación parenteral. Evolucionó en coma, con signos de shock, hepatomegalia, deposiciones liquidas sanguinolentas y sangramiento por los orificios de punción. Falleció 21 horas después de ingresar mientras se intentaba denudación venosa. La noche del ingreso, el niño había dormido con su madre, cubierto con 3 frazadas y una cubrecama.

2. Lactante de 5 meses, masculino, desnutrido grado Il, con antecedentes de una hospitalización reciente por diarrea aguda y deshidratación. Dado de alta el día anterior a su segunda hospi- talización, reingresó por convulsiones y fiebre alta desde 2 horas antes, en coma, con convulsiones generalizadas, temperatura rectal $42^{\dot{\alpha}} \mathrm{C}$, hidratado, fontanela a tensión normal, sin signos aparentes de infección localizada. Se trató con fenobarbital $10 \mathrm{mg} \mathrm{x} \mathrm{kg} \mathrm{i.m.;} \mathrm{paños} \mathrm{fríos,} \mathrm{ampi-}$ cilina $100 \mathrm{mg} \times \mathrm{kg} x$ día e.v., gentamicina $6 \mathrm{mg} \mathrm{x}$ $\mathrm{kg} x$ día i.m., hidratación parenteral con solución de glucosa $5 \%$ y $70 \mathrm{~m} \mathrm{Eq} \times \mathrm{L}$ de $\mathrm{Na}+, 100 \mathrm{ml} \times$ $\mathrm{kg}$, en las primeras 12 horas. Evolucionó sin signes de recuperación de conciencia, con hepatomegalia progresiva, convulsiones a repetición, deposiciones líquidas, hemorragia digestiva alta, fenómenos hemorragiparos y shock. Falleció a los 7 dias de hospitalización. La madre dio el antecedente de haber dormido con el niño la noche de su ingreso, abrigada con 3 frazadas y con un brasero encendido en la pieza.

3. Lactante de 1 mes 8 dias, masculino, ingresó a las 2,30 AM, muy grave, con hipertermia de $42,1^{\circ} \mathrm{C}$ rectal, inconciente, cianótico, respiración profunda, subcrépitos bilaterales, hígado a $1 \mathrm{~cm}$, deposiciones liquidas $y$ herida sangrante perianal, hidratado, con bregma a tensión normal. Diez dias antes habia presentado tos y quejido, recibió ampicilina por 2 días, luego penicilina sódica in1ramuscular por 3 días, la madre no completó el tratamiento indicado. Desde su ingreso se mantuvo en coma profundo, con con-

Tabla 1.

Exámenes de laboratorio

\begin{tabular}{|c|c|c|c|c|}
\hline & Caso 1 & Caso 2 & Caso 3 & Caso 4 \\
\hline Sodia $\mathrm{m} \mathrm{Eq} \times 1$ & 150 & 144 & 137 & 148 \\
\hline Potasio m Eq $\times 1$ & 4,8 & 5.3 & 5,2 & 5 \\
\hline $\mathrm{Ph}$ & 7,29 & 7,10 & 7,23 & 7,4 \\
\hline Glicemia g $\%$ & 2 & $\mathrm{NR}$ & NR & 0,28 \\
\hline Fosfatasas alcalinas & 766 & 179 & NR & NR \\
\hline Tiempo trotrombina $\%$ & NR & 49 & no coagula & 71 \\
\hline P. TT & $\mathrm{NR}$ & $\mathrm{NR}$ & $147^{\prime \prime}$ & NR \\
\hline Transaminta sas (CPT) & NR & 28 & NR & 67 \\
\hline Creatininemia & 1,68 & 0,83 & NR & 0,80 \\
\hline Uremiag $/ / 00$ & 0,48 & NR & NR & 0,60 \\
\hline Calcemia mg \% & NR & 5,55 & NR & NR \\
\hline Amonemia mg \% & NR & 19,6 & NR & 58,8 \\
\hline Leucocitos $\mathrm{mm}^{3}$ & 24.800 & 16.000 & 22.000 & 17.500 \\
\hline Hemoglobina g \% & 14,5 & 13,8 & 14,4 & 10,5 \\
\hline L.C.R. Glucosa g \%/oo & 1,25 & 0,70 & 0,53 & 0,25 \\
\hline Proteina $9 \%$ & 0,10 & 0.52 & NR & 0,15 \\
\hline Leucocitos & 4 & 2 & NR & 3 \\
\hline Cultivo LCR & $(-)$ & $(-)$ & $(-)$ & $(-)$ \\
\hline Hemocultivo & $(-)$ & $(-)$ & $(-)$ & $(-)$ \\
\hline Coprocultivo & $(-)$ & $(-)$ & NR & $(-)$ \\
\hline Fibrinógeno mg $\%$ & $N R$ & NR & 29 & \\
\hline Tomografia axial (TAC) & NR. & $\mathrm{NR}$ & NR & normal \\
\hline Encefalograma & NR & NR & NR & $\begin{array}{l}\text { sufrimiento } \\
\text { neuroral } \\
\text { hemisferio izdo. }\end{array}$ \\
\hline
\end{tabular}

NR = no realizado.

$(-)=$. negativo 
vulsiones generalizadas, posición de decorticación, hipertonía, hiperreflexia, rédceión de Babinski bilateral espontánea, fenómenos hemorágicos en piel y mucosas, hepatomegalia progresiva. Fue tratado con fenobarbital $10 \mathrm{mg} x \mathrm{~kg}$ i.m., ampicilina $100 \mathrm{mg} \times \mathrm{kg} x$ dia e.v., gentamicina $7,5 \mathrm{mg} \times \mathrm{kg} x$ día i.m., e hidratación parenteral con solución de glucosa $5 \%$ y bicarbonato de sodio $35 \mathrm{~m} \mathrm{Eq} \times \mathrm{L}, 100 \mathrm{ml} \times \mathrm{kg}$ en las primeras 12 horas. Posteriormente recibió crioprecipitado de plasma. Fallecio 19 horas despues de su ingreso.

4. Lactante de 3 meses, masculino, con antecedentes de deposiciones alteradas de l dia de evolución, ingresó con temperatura rectal de $39,5^{\circ} \mathrm{C}$, convulsiones, hipertonia y compromiso de conciencia. Evolucionó grave, soporoso, con convulsiones tónico-clónicas generaljzadas de dificil manejo, diarrea, melena, fenómenos hemorrágicos cutáneos y hepatomegalia de $2 \mathrm{~cm}$ bajo el reborde costal. Recibio tratamiento con fenobarbital $10 \mathrm{mg} x \mathrm{~kg}$ j.m., ampicilina $100 \mathrm{mg}$ $\mathrm{x} \mathrm{kg} \times \mathrm{d}$ dia e.v., gentamicina $5 \mathrm{mg} \mathrm{x} \mathrm{kg} \mathrm{x}$ día i.m., e hidratación parenteral con solución de glucosa $5 \%$, con $70 \mathrm{~m} \mathrm{Eq} \times \mathrm{L}$ de $\mathrm{Na}+, 150 \mathrm{ml} \times \mathrm{kg} \mathrm{en}$ las primeras 24 horas. Después de 8 dias en coma profundo, recuperó paulatinamente la conciencia. Fue dado de alta a los 25 días después del ingreso, con deterioro de desarrollo psicomotor, posición de decorticación, hemiparesia doble espástica y nistagmus.

Los exámenes de laboratorio de los 4 casos están resurnidos en la Tabla 1 .

En los tres fallecidos, se practicó necropsia; los hallazgos más característicos fueron edema cerebral intenso, lesiones neuronales, necrosis acidófila extensa de células nerviosas, acentuada tumefacción de los astrocitos con citoplasma acuoso y gliosis focal y en uno de ellos evidencias de coagulación intra vascular (figura 1).

En el higado la arquitectura estaba conservada, había hiperemia y leve a moderada infiltración grasa focal en grandes vacuolas que ocupaban parte de la célula. Algunos hepatocitos presentaban pigmento biliar en el citoplasma (figura 2).

Habia edema e hiperemia de la mucosa intestinal, en el exámen microscópico, lámina propia edematosa con infiltrado inflamatorio de linfocitos $y$ plasmacélulas, nódulos linfáticos grandes, muy celulares, células centrocíticas, epiteloídeas, con citoplasma acidófilo abundante, con necrosis de los blastos del centro germinal y fagocitosis de restos celulares (figura 3 ).

La médula renal estaba hiperémica, tenía tumefacción turbia de las células epiteliales e infiltrado intersticial con células redondas $y$ habian pequeños focos hemorrágicos medulares.

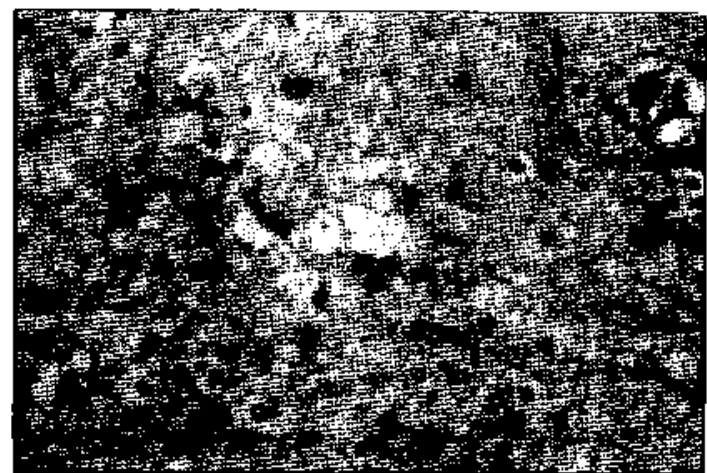

Figura J: Microlotogralia del cetebro: gliosis con acentuad tu tuefacción de los astrocitos.

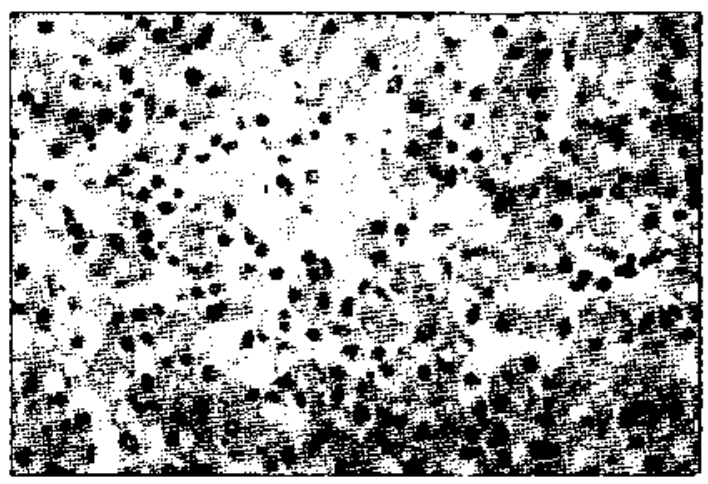

Figura 2: Micrototografía del hígado infïtración grasa celular con grandes vacuolas-

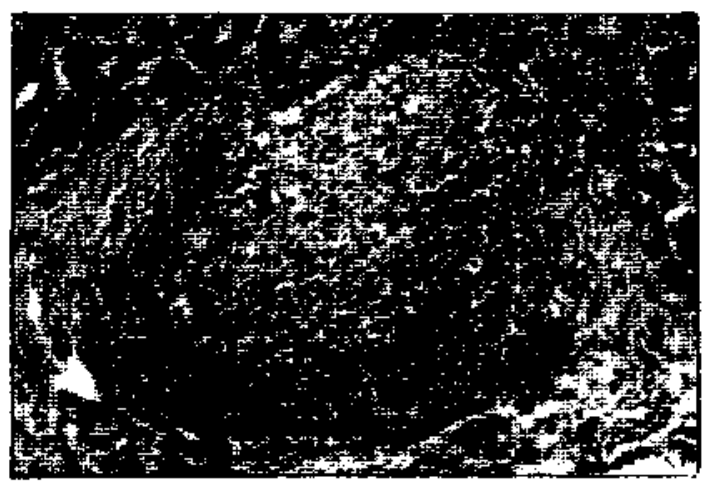

Figura 3: Microfotografía de intestino: nódulo lintático muy celular con necrosis de los blastos del centro germinal.

Bazo hiperémico en la médula con hiperplasia de pulpa blanca, folículos linfáticos prominentes con centros germinales necróticos y fagocitosis. Se observa disminución de la población de linfocitos. Hiperemia moderada a intensa en el pulmón. En el miocardio tumefacción turbia de las células. Acentuada disminución de los linfocitos de la corteza del timo y abundantes macrófagos con linfofagocitos. 


\section{DISCUSION}

Los pacientes descritos muestran casi todos los hechos que caracterizan el golpe de calor ${ }^{3}$. La septicemia, el síndrome de Reye, las convulsiones prolongadas y la intoxicación por drogas fueron razonablemente descartadas aunque en dos pacientes no fue posible indagar sobre maniobras exageradas de aislamiento térmico, en los otros dos los antecedentes fueron claros en tal sentido.

En 1983 se describió en lactantes menores de un año un sindrome caracterizado por encefalopatía de comienzo brusco, fiebre, shock, depusiciones liquidas, coagulación intravascular dise. minada, acidosis y alteraciones de las funciones renal y hepática, de gran letalidad y con severas secuelas neurológicas en los sobrevivientes ${ }^{4}$. Posteriomente han aparecido numerosos casos similares $5,6,9,10$ bajo el denominador común de shock hemorrágico y encefalopatia: el análisis clínico, patológico y de laboratorio muestra que constituyen una sola entidad con el golpe de calor 11,12 .

Se cree que los lactantes son particularmente susceptibles al golpe de calor por la labilidad térmica que los caracteriza, su elevado metabolismo basal y su incapacidad para librarse del exceso de abrigo ${ }^{7}$. En los menores de un año hay cierto desbalance en la termoregulación pues la producción de calor por superficie corporal alcanza el máxino a la edad de 5 meses, en cambio la capacidad para disiparlo por evaporación (sudoración) se desarrolla más lentamente a lo largo del primer año.

Se acostumbra abrigar excesivamente a los lactantes, especialmente si están enfermos: se sabe que cubriendo enteramente a un adulto con material sintético, su temperatura se eleva en $1^{\circ} \mathrm{C}$ por hora, $y$ es probable que el alza sea mayor en los lactantes. Aunque no se sabe cuanto tiempo es necesario para provocar el golpe de calor, en un caso, bastaron 30 minutos en el interior de un automovil al sol, pará causarlo.

Es probable que el golpe de calor ocurra en una amplia gama de intensidad, desde casos que se recuperan totalmente, hasta otros fulminantes que causan la muerte en pocas horas; desgraciadamente aún no conocemos índices que nos permitan saber anticipadamente la evolución de cada paciente, pero la mayor edad podría ser un índice favorable.

En un total de 22 pacientes, de diferentes orígenes, nos ha llamado mucho la atención, el predominio notable del sexo mascultno (19 varones) pero no sabemos si el sexo masculino es menos resistente a la hipertermia o si existe otra explicación para el hecho.

Las lesiones anatomopatológicas descritas por
Bacon $^{7}$ son similares a las encontradas por nosotros; todas son inespecificas y corresponden a un daño agudo que puede ser de diversa naturaleza. Coincidimos con ęl mismo autor en que la hiper. termia es la causa de este cuadro catastrófico en lactantes, por su semejanza con el golpe de calor descrito en adultos y niños mayores.

La educación de las madres, sobre el peligro de abrigar excesivamente a los lactantes, especialmente si tienen fiebre, podría prevenir estos casos tan lamentables.

El golpe de calor, debe tenerse en cuenta en pacientes con encefalopatia aguda e bipertermia. y debe tratarse rápidamente bajando la temperatura con medios físicos, administración de liqujdos endovenosos, oxígeno y combatiendo el edema cerebral.

\section{RESUMEN}

Se presentan 4 casos en lactantes de encefa. lopatía aguda, con hipertermia, shock y fenómenos hemorragiparos. Se describe el cuadro clínico, de laboratorio y anatomopatológico. señalándose su predominio en el sexo masculino y las lesiones anatomopatológicas más características.

\section{REFERENCIAS}

1. Bacon, C., Scott, D., Jones P.: Heatstroke in wellwrapped infants. Lancet l: $422,1979$.

2. Laplane, R.: Coup de chaleur hivesnal. Le Nourrison 33: 50, 1945 .

3. Bacon, C., Beliman, M.: Heatstroke as a possible cause of encephalopathy. Bt Med J 287: 328. 1983.

4. Levin, $M .:$ Hetnorrhagic shock and encephalopathy: A new syndrome with a high morthality in young children. Lancet 2: 64, 1979.

5. Lafeber, H.N. Voort, E., De Groot, R.: Hemorrhagic shock and encephalopathy syndrome. Lancet 2 : 395. 1983.

6. Whittington. L., Roscelli, J., Porry. W.H.: Hemorrhagic shock and encephalopathy: Forther description of a new syndrome. J Pediatt 106: 599. 1985 .

7. Bacon, C.: Overheating in infancy, Arch Dis Child 58: 673, 1983 .

8. Shibolet, S.: Heatstroke: its chinieal picture and mechanism in 36 cases. J Med 36:525, 1967.

9. Phts communicable Diseose Surveillance Centre. Survellance Scheme for hemorrhagic shock encephalopathy syndrome: surveillance report for 1982-1984. Br Med J 290: 1578, 1985.

10. Ross, R., Sullivan, 1., Matchew, D.: Hemorrhagic shock encephalopathic syndrome and neurodevelopment delay. Lancet 2: 441, 1985.

11. Editorial: Hemorrhagic shock and encephalopathy. lancet 2: 534,1985 .

12. Stanion. A.N.: Hemorrhagic and encephalopathy, Lancet 2: 1019, 1985. 